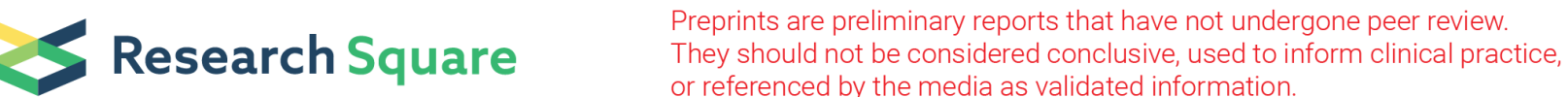

\section{Percutaneous Mastoid Electrical Stimulator improves poststroke depression and cognitive function in patients with ischemic stroke}

Taoli Lu

The Second People's Hospital of Chengdu

lanying he ( $031324679 @ q q . c o m$ )

Second people's Hospital of Chengdu https://orcid.org/0000-0002-7127-6333

Bei Zhang

The Second People's Hospital of Chengdu

Jian Wang

The Second People's Hospital of Chengdu

Lili Zhang

The Second People's Hospital of Chengdu

\section{WeiWei Dong}

Chongqing Medical University Affiliated Children's Hospital

Hao Yang

Chongqing University

\section{Research article}

Keywords: acute ischemic stroke, percutaneous mastoid electrical stimulator, cognition, poststroke depression

Posted Date: November 26th, 2019

DOI: https://doi.org/10.21203/rs.2.17451/v1

License: (c) (1) This work is licensed under a Creative Commons Attribution 4.0 International License. Read Full License 


\section{Abstract}

Backgrond Poststroke depression could lead to functional dependence, cognitive impairment and reduced quality of life. The aim of this study was to evaluate the effects of percutaneous mastoid electrical stimulator (PMES) plus antidepressant on poststroke depression and cognitive function.

Methods 258 clinically depressed ischemic stroke patients within $14 \mathrm{~d}$ of index stroke were randomly assigned to PMES plus antidepressant (PMES group) and sham plus antidepressant (sham group). All patients underwent Montreal Cognitive Assessment (MoCA) and Hamilton Rating Scale for Depression (HRSD) test at 2 weeks (baseline), and 6 months after the stroke. The clinical outcome was defined as treatment response ( $\geq 50 \%$ reduction in HRSD) and depression remission (HRSD $\leq 9)$ at 6 months.

Results Compared with the sham group, PEMS group significantly improved MoCA and HRSD scoreat the end of the 6-month treatment period $(P<0.001)$. The mean value of MoCA score change $(3.50 \pm 2.55 \mathrm{vs}$ $2.72 \pm 2.52 ; P=0.005)$, and HRSD score change $(-11.93 \pm 5.32$ vs $-10.48 \pm 6.10, P=0.041)$ was significantly greater in the PMES group compared to sham group at 6 months. In fully adjusted models, PMES plus antidepressant was associated with increased treatment response rate [adjusted odds ratio (OR), 2.04; 95\% confidence interval $(\mathrm{Cl}), 1.15-3.63 ; \mathrm{P}=0.015$ ], and depression remission rate [adjusted $\mathrm{OR}, 1.83 ; 95 \%$ $\mathrm{Cl}, 1.05-3.18 ; \mathrm{P}=0.032$ ]. In fully adjust models, $\mathrm{PMES}$ plus antidepressant was associated with decreased risk of 6-month MoCA score <26 (adjusted OR, 0.25; 95\%Cl, 0.09-0.70; $\mathrm{P}=0.008$ ).

Conclusion These findings demonstrate that PMES adjunctive to antidepressant therapy is highly effective in reducing depression, achieving remission in the short term, and improving cognition.

\section{Background}

Stroke is a leading cause of long-term disability. Despite of impressive progress in early diagnosis and medical treatment which result in the decrease of incidence and mortality rates of stroke,

about $25 \%-74 \%$ stroke patients still suffer major disability and psychological illness, including depression, cognitive impairment, and social isolation[1,2]. Poststroke depression (PSD) is associated with poor outcomes after stroke, including cognitive disorders, poor rehabilitation outcomes[3,4]. PSD has a prevalence of about $30 \%$ in stroke survivors based on the previous studies[5].

In 1998, neuroprotection of fastigial nucleus stimulation (FNS) was first confirmed by Reis at al [6]. FNS has a variety of neuroprotective mechanisms. It can inhibit the electrical activity around the focus, reduce the excitotoxic injury of neurons, inhibit the inflammatory response, and inhibit apoptosis [7]. In addition, FNS has been reported to improve cognitive impairment and depression after stroke in animal experiments [8-10].

The non-invasive percutaneous mastoid electrical stimulator (PMES) can simulate experimental FNS and has been used in the clinical treatment of stroke patients [7]. Some observation studies show that PMES 
treatment can improve the clinical prognosis and show good safety [11-14]. The purpose of this study was to explore the effect of PMES combined with antidepressants on post-stroke depression and cognitive function.

\section{Methods}

\section{Study Population}

This study was a prospective, randomized, double-blind, and sham-controlled study. This project was registered in the Chinese Clinical Trial Register (ChiCTR) (registration number: ChiCTR-ICR-15006273) and was performed according to the CONSORT 2010 extension to randomised pilot and feasibility trials [15]. The patients were admitted to the Second People's Hospital of Chengdu due to ischemic stroke within 2 weeks of symptom onset between January 2015 and December 2018. All patient with a clinical diagnosis of ischemic stroke confirmed by brain computed tomography or magnetic resonance imaging.

\section{Depression Screening}

The diagnosis of clinical depression was verified by using DSM V criteria. Depression screening was carried out by 30 -item Geriatric Depression Scale(GDS), which consists of 30 questions, are scored as 1 point individually, resulting in 0-30 points and being classified as: $0-10$, no depression; 11-20, mild depression; 21-30, moderate depression. The diagnosis of depression was validated by Hamilton Rating Scale for Depression (HRSD) in those who scored $\geq 11$ on the GDS and consented to the full study. Stoke severity was assessed based on National Institutes of Health Stroke Scale (NIHSS). The study was approved by the ethics committees of the medical centers. Informed consent was signed by all the participants.

\section{Inclusion and exclusion criteria}

Patients were included if they fulfilled all the following criteria: (1) Admission for first-ever ischemic stroke within 14d, (2)no neurological or psychiatric disease before stroke, (3) no aphasia,(4) no drug, (5) no hearing deficit, (6) right-handed, (7) Finnish-speaking and (8) able to co-operate,(9) no active malignancies;(10) patients could appropriately communicate.

\section{Study Design and grouping}

Patients were divided into two groups: the sham and PMES groups. The patients in PMES group received PMES treatment as an add-on to antidepressant and the patients in sham group received sham stimulation and antidepressant.

\section{Treatment methods}

After cleaning the bilateral mastoid skin behind the ears, stimulation electrodes were placed. The electrode size was $42 \times 24 \mathrm{~mm}$ and the conductive area was $19 \mathrm{~mm}$ (Figure 1). The stimulation parameters: 
the pulse width of PMES and sham was $9 \mu \mathrm{S}$, the pulse frequency of PMES and sham was $1.8 \mathrm{khz}, 10 \mathrm{~Hz}$, respectively. The peak current of PMES and sham was $10 \mathrm{~mA}$ and $0.18 \mathrm{~mA}$, respectively. Previous studies have shown that $10 \mathrm{~mA}$ is very safe, and some patients may have slight tingling, but no skin redness or burn [16]. In order to reduce the surface sensation caused by current stimulation, we modulated the lowfrequency signal (13-45hz) to the intermediate frequency signal of $1.8 \mathrm{kHz}$ and set $1.0-1.2 \mathrm{v}$ as the voltage range of the low-frequency signal. This change in the modulation signal in this range causes a slight squeeze. The intermediate-frequency signal was the exponential decay signal with a base of "a" $(0<a<1)$. The signal was a non-polar exponential waveform, which was composed of positive and negative pulse waves and equivalent charges. Negative pulse can depolarize the nerve fiber, while positive pulse can balance the charge, thus eliminating the accumulation of electrostatic charge and reducing the adverse electrochemical reaction. In order to reduce the energy of single pulse, we reduce the base value "a", not the pulse width, thus reducing the degree of extrusion. The surface sensation of real stimulus was close to the surface sensation of sham stimulus, which was a periodic point-contact sense of touch. PMES group and sham group were treated 45 minutes/day lasted 3 months.

In this study, selective serotonin reuptake inhibitor (SSRI) is recommended as the first choice for depressive patients, and sertraline is recommended as the initial antidepressant because of its tolerance to medical treatment and relatively low incidence of cardiovascular side effects.

\section{Randomization and double blinding}

Patients who met the criteria were assigned to treatment groups according to a predefined randomization plan by using a block size of 4 , a ratio of $1: 1$, and stratified by study center. Patients, investigators and all study personnel were blinded to the treatment allocation. The PMES and sham stimulators had the same external appearances, user manuals and electrodes. They could not be distinguished by their external appearance without a detection device. We took the following measures to guarantee double-blinding: enrolled patients were not acquainted with each other, there was no physical contact or communication (such as sensory perception) between patients during visits, and all of the patients would be told when enrolled that it was not possible to accurately judge whether they were receiving true or sham stimulation only based on the surface sensation.

\section{Data Collection}

Baseline characteristics including demographics, stroke characteristics, NIHSS, risk factors. All patients underwent cognitive and depressive state assessment at 2 weeks (baseline) and 6 months after stroke.

Cognitive state was assessed using the Montreal Cognitive Assessment (MoCA), scores range from 0 to 30 points, with a lower score reflecting greater cognitive impairment, and a cut-off of $<26$ was considered as indicative of cognitive impairment. Depressive state was assessed using HRSD. Treatment response was defined as $\geq 50 \%$ reduction in HRSD. Remission has been defined variably as HRSD score of $\leq 9$ (no longer meeting depression criterion), $\leq 7$ (absence of any depressive symptoms), or $\leq 3$ (equivalent to 
healthy controls). We used HRSD score of $\leq 9$ and $\geq 50 \%$ reduction in HRSD for comparison with baseline.

All patients were followed up for 6 months. The change in HRSD and MoCA was detected at 6 months after treatment. The clinical outcome was defined as treatment response( $\geq 50 \%$ reduction in HRSD) and depression remission (HRSD $\leq 9)$ at 6 months after stroke onset.

\section{Statistical analysis}

Firstly, patients were classified into sham and PMES groups. Demographic characteristics, vascular risk factors, current smoking, and so on were compared between the 2 subgroups in univariate analysis, using Pearson $\chi 2$ test, Fisher exact 2-sided test, or Student $t$ test, mean values( \pm standard deviation) were calculated for continuous variables. Mann-Whitney $U$ test was used to test differences between two groups. Secondly, We then performed logistic regressions analyses to determine the association between PMES and treatment response, depression remission and MoCA $<26$, adjusting for all confounders (age, NIHSS, sex, BMI区hypertension, current smoking, current drinking, diabetes, hyperlipidemia, atrial fibrillation, family history of stroke, baseline MoCA score or 6-month MoCA score, HRSD score, medications use, infarct location). Results were expressed as adjusted odds ratios (OR) with the corresponding 95\% confidence interval (Cl). The data were analyzed using SPSS software (SPASS 22.0). $P$ values $<0.05$ were considered as statistically significant.

\section{Result}

\section{Characteristics of the study subjects}

A total of 288 patients were enrolled in this study (sham group, $\mathrm{N}=144$; PMES group, $\mathrm{N}=144$ ). 12 patients were lost to follow-up after discharge from the hospital (sham group, $\mathrm{N}=3$; PMES group, $\mathrm{N}=9$ ), recurrent stroke occurred in 10 patients(sham group, $N=4$; PMES group, $N=6$ ), 8 patients died within 6 months(sham group, $\mathrm{N}=4$; PMES group, $\mathrm{N}=4$ ); . Thus, a total of 258 patients (sham group, $\mathrm{N}=133$; PMES group, $\mathrm{N}=125$ ) comprised the data as the available analysis set, comprised $52.33 \%$ (135) men and $47.67 \%$ (123) women, the mean age was $65.58 \pm 8.59$ years(range:42-87 years). In the study population, 148 patients had a history of hypertension, 97 had a history of diabetes, 139 had a history of hyperlipidemia, 91 patients smoke. The PMES and sham groups received treatment daily for 45 minutes, and the treatment lasted 6 months. There were no adverse reactions reported either in the PMES group or in the sham group during the treatment period. No patients stopped taking antidepressant during the follow-up period.

Baseline characteristics of patients in the sham group and the PMES group were compared (Table 1). There were also no significant group differences in the baseline HRSD and MoCA score (P凶0.05).

\section{PMES significantly improves MoCA Score and HRSD Score}


There was no difference in MOCA score, HRSD score at baseline, compared with the baseline, the percentage of MoCA score <26 was not different between PEMS and sham groups(72/125 vs73/133,P=0.661). MoCA score and HRSD score of the 6-month treatment period improved both in sham and PMES groups, the percentage MoCA score <26 in PEMS group was lower than that in sham group(15/125 vs 32/133, $P=0.012$ ), MoCA score in PMES group was higher than that in sham group at 6 month (28.26 \pm 1.95 vs $27.26 \pm 2.20, P=0.000$ ), in addition, the mean value of MoCA score change (M6baseline) was higher in PMES group(3.50 \pm 2.55$)$ compared to sham group $(2.72 \pm 2.52 ; P=0.005)$. After adjusting for all confounders, PMES was associated with decreased risk of 6-month MoCA score $<26$ (adjusted OR, $0.25 ; 95 \% \mathrm{Cl}, 0.09-0.70 ; P=0.008)$. HRSD score was lower in PEMS (9.58 \pm 3.45 vs $11.54 \pm 4.21, P=0.000$ ), mean decrease in HRSD in the PEMS group was significantly greater at 6 months when compared with the sham group $(-11.93 \pm 5.32$ versus $-10.48 \pm 6.10, P=0.041)$ (Table 2).

\section{Multivariable Models on the Association between PEMS and poststroke depression}

During the 6-month follow-up period, 126 patients had treatment response, 94 patients had depression remission. Treatment response in sham group were $41.35 \%(55 / 133)$ at 6 months, which was significantly lower than that in the PMES group $(56.8 \%, 71 / 125)(P=0.013)$.After adjusting for age, NIHSS, sex, BMIX hypertension, current smoking, current drinking, diabetes, hyperlipidemia, atrial fibrillation, family history of stroke, baseline MoCA score, HRSD score, medications use, infarct location(Model 1), PMES was associated with increased treatment response rate (adjusted $\mathrm{OR}, 2.04 ; 95 \% \mathrm{Cl}, 1.15-3.63 ; \mathrm{P}=0.015$ ), even when 6-month MoCA score were entered into multivariate logistic regression (Model 2), PMES resulted to be associated with increased treatment response rate (adjusted $\mathrm{OR}, 2.12 ; 95 \% \mathrm{Cl}, 1.17-3.86 ; \mathrm{P}=0.013$ ).

Depression remission in sham group were 29.32\%(39/133) at 6 months, which was significantly lower than that in the PMES group $(44.00 \%, 55 / 125)(P=0.014)$. In fully adjusted models(Model 1$)$, PMES was associated with depression remission rate (adjusted $\mathrm{OR}, 1.83 ; 95 \% \mathrm{Cl}, 1.05-3.18$; $\mathrm{P}=0.032$ ), when 6month MoCA score were entered into multivariate logistic regression(Model 2), PMES resulted to be associated with increased depression remission rate (adjusted $\mathrm{OR}, 1.70 ; 95 \% \mathrm{Cl}, 0.94-3.06 ; \mathrm{P}=0.047$ ) (Table 3).

\section{Discussion}

The incidence of PSD is very high. PSD affects $12 \%-72 \%$ of stroke patients $[17,18]$. A meta-analysis showed that $31 \%$ of patients developed depression within 5 years after stroke. In the past, physical disablity caused by stroke was often the focus of treatment. However, in recent years, the treatment of psychological comorbidities has also attracted the attention of clinicians, which affected the effect of patients' rehabilitation. The purpose of this study is to report the efficacy of a combination of non drug therapy and drug therapy for PSD. Noninvasive transcutaneous FNS device is called CVFT device in China, uses biomimetic current to simulate FNS and has been used in China for many years. Its safety has been confirmed in clinical research. 
We observed that PMES improved the emotion and cognition in stroke patients. In previous animal studies, it was found that FNS could up-regulated noradrenaline (NE) and 5-HT in the frontal lobe of depressed rats $[19,20]$. Recent clinical studies have shown that FNS alone or in combination with drug therapy can improve PSD [21]. Fan et al. found that the cognitive function decreased on 2 months after chronic cerebral hypoperfusion, and was worse on 4 months after hypoperfusion, the cognitive function improved after FNS treatment [10]. These data suggest that FNS may have potential in the treatment of patients with vascular or poststroke cognitive impairment.

PMES combined with antidepressant has been significantly more successful in improving poststroke depression than medication alone. In this study, during the 6-month follow-up period, the higher percentage of patients having a HRSD score of $\leq 9$ and $\geq 50 \%$ reduction in the PMES group compared to the sham group showed that more patients from the PMES group regained less depressed and confused mood. In addition, the mean value of MoCA score change was higher in PMES group compared to sham group, and the percentage MoCA score < 26 in PEMS group was lower than that in sham group. In fully adjusted models, there was an association between PMES and improved poststroke depression and cognition. The study showed that daily treatment with PMES was effective in poststroke depression and cognitive impairment.

The exact mechanism of action of PMES is unclear. According to previous studies, FNS could up-regulate $\mathrm{NE}$ and $5-\mathrm{HT}$ in the frontal lobes of rats with depression[19,20], in additional, the positive affective state or enhanced arousal and attention could improve cognition, which seems a plausible mechanism.

\section{Limitations}

Some limitations of this study merit consideration. Firstly, NIHSS score has been shown to correlate with infarction volume, we lacked data on infarction volume. Secondly, peak current is $10 \mathrm{~mA}$ for PMES and $0.18 \mathrm{~mA}$ for sham, which might give patients clue about group assignment and have an effect on the experimental results. Thirdly, each group were prescribed and reported taking antidepressants during the 6 months treatment period, the doses and type of drug were not standardized. This is a limitation of the study but represents the context of everyday practice.

\section{Conclusions}

In conclusion, our findings indicated that PMES adjunctive to antidepressant therapy is highly effective in reducing depression and achieving remission in the short term. We also demonstrated that improved poststroke depressive was associated with improved cognition. These data indicate that PMES may be a safe and low-cost therapy to improve clinical stroke outcomes.

\section{Declarations}

\section{Abbreviations}


percutaneous mastoid electrical stimulator (PMES) Hamilton Rating Scale for Depression (HRSD) Montreal Cognitive Assessment (MoCA) Poststroke depression (PSD) Fastigial nucleus stimulation cerebrovascular function therapy (CVFT) National Institutes of Health Stroke Scale

\section{Ethics approval and consent to participate}

We obtained ethical approval for this study from the Medical and Health Research Ethics Committee at Second people's Hospital of Chengdu. The current study was carried out according to Declaration of Helsinki. Written informed consent was obtained from all study participants or their legal proxies.

\section{Consent for publication}

Not applicable.

\section{Availability of data and materials}

Data used in this study may be available by request to corresponding author via email: 531324679@qq.com

\section{Competing interests}

The authors declare that they have no competing interests.

\section{Funding}

This work was funded by the Health and Family Planning Commission of Chengdu (2015009). The funding body did not participate in designing the study or writing the manuscript. The study protocol has undergone peer-review process by the funding body.

\section{Authors' contributions}

TLL was responsible for the data collection and analysis and the first draft of the paper and further manuscript. LYH was responsible for the concept and design of the study.BZ was responsible for the data collection and analysis. JW was responsible for the design of the study.LLZ was responsible for the data analysis, and interpretation.WWD was responsible for the interpretation. HY was responsible for the data analysis All authors read and approved the final manuscript for publication.

\section{Acknowledgments}

The devices were provided by Chongqing Haikun Medical Instrument Co., Ltd. None of the investigators has any financial interest in Chongqing Haikun Medical Instrument Co., Ltd.

\section{References}


1. Stein LA, Goldmann E, Zamzam A, Luciano JM, Messé SR, Cucchiara BL, Kasner SE, Mullen MT.Association Between Anxiety, Depression, and Post-traumatic Stress Disorder and Outcomes After Ischemic Stroke. Front Neurol.2018;9:890. Doi: 10.3389/fneur.2018.00890. eCollection 2018

2. Villa RF, Ferrari F, Moretti A. Post-stroke depression: Mechanisms and pharmacological treatment. Pharmacol Ther. 2018;184:131-144. Doi: 10.1016/j.pharmthera.2017.11.005.

3. Love MF, Sharrief A, Chaoul A, Savitz S, Beauchamp JES.Mind-Body Interventions, Psychological Stressors, and Quality of Life in Stroke Survivors.Stroke. 2019;50:434-440. Doi: 10.1161/STROKEAHA.118.021150.

4. Huang J, Zhou FC, Guan B, Zhang N, Wang A, Yu P, Zhou L, Wang CY, Wang C. Predictors of Remission of Early-Onset Poststroke Depression and the Interaction Between Depression and Cognition During Follow-Up. Front Psychiatry. 2019;9:738. Doi: 10.3389/fpsyt.2018.00738. eCollection 2018.

5. De RA, Fransen E, Brouns R, et al. Poststroke depression and its multifactorial nature: results from a prospective longitudinal study. J Neurol Sci.2014;347:159-166.Doi: 10.1016/j.jns.2014.09.038.

6. Nedergaard M, Hansen AJ. Characterization of cortical depolarizations evoked in focal cerebral ischemia. J Cereb Blood Flow Metab.1993;13:568-574.DOI:10.1038/jcbfm.1993.74

7. Wang J, Dong WW, Zhang WH, Zheng J, Wang X. Electrical stimulation of cerebellar fastigial nucleus: mechanism of neuroprotection and prospects for clinical application against cerebral ischemia. CNS. Neurosci Ther. 2014;20:710-716. Doi: 10.1111/cns.12288.

8. Sui R, Zhang L, Min L, Yuan J, Li X. Cerebellar dysfunction may play an important role in poststroke depression. Med Hypotheses 2009;72:643-646.Doi: 10.1016/j.mehy.2008.11.042

9. Baldacara L, Borgio JG, Lacerda AL, Jackowski AP. Cerebellum and psychiatric disorders. Rev Bras Psiquiatr 2008;30:281-289

10. Tan XL, Dong WW, Yang QD. The prophylactic and curative effects of electrical stimulation of cerebellar fastigial nucleus on vascular dementia in rats. Stroke Nerv Dis 2004;11:349-352.

11. Niu LC, Li T, Lei JA, Han Q, Li YX, Zhang BQ, Yan PJ. Effect of cerebellar fastigial nucleus treated by electrical stimulation on 70 patiengts with acute cerebral infarction. Chin J Rehabil Theory Practice.2005;11:795-796.

12. Xu X. Application of cerebellar fustigial nucleus electric stimulation on rehabilitation of cerebrovascular diseases. Lab Med Clin,2010;7:1318-1321.

13. Shen HQ. Cerebral circulation function therapeutic apparatus of the role of the mechanism and clinical effect. Prog Biomed Eng.2009;30:245-246. 
14. He WY, Li BP, Huang XZ. Clinical observation of treatment of acute cerebral infarction by electrical stimulation of the fastigial nucleus. J Apoplexy Nerv Dis.2003;20:470.

15. Eldridge SM, Chan CL, Campbell MJ, Bond CM, Hopewell S, Thabane L,Lancaster GA. group Pc: CONSORT 2010 statement: extension to randomised pilot and feasibility trials. BMJ. 2016;355:i5239.

16. Juan $Y$, Shu O, Jinhe L, Na Y, Yushuang D, Weiwei D, Lanying H, Jian W. Migraine prevention with percutaneous mastoid electrical stimulator: A randomized double-blind controlled trial. Cephalalgia.2017;37:1248-1256. Doi: 10.1177/0333102416678623

17. Paolucci S. Epidemiology and treatment of post-stroke depression. Neuropsychiatr Dis Treat2008;4:145-117.

18. Torrisi M, De Cola MC, Buda A, Carioti L, Scaltrito MV, Bramanti P, Manuli A, De Luca R, Calabrò RS. Self-Efficacy, Poststroke Depression, and Rehabilitation Outcomes: Is There a Correlation?J Stroke Cerebrovasc Dis. 2018;27(11):3208-3211. Doi:

10.1016/j.jstrokecerebrovasdis.2018.07.021.

19. Gao W, Wang N, Qiao H.The effects of 5-HT1B receptor subtypes on motor behaviors mediated by cerebellar fastigial nucleus. Zhongguo Ying Yong Sheng Li Xue Za Zhi. 2016;32:550-554. Doi: 10.13459/j.cnki.cjap.2016.06.014

20. Zhang Runfeng,Li Xia,Chen Yun zhen, Tang Ke xin, Ma Guo-zhong, Huang Xuan yin. Lu0 2aigui4. Feng Jin. Effects of cerebellar fastigial nucleus electro-stimulation on release of neurotransmitters in ischemic hearts.Chinese Journal of Clinical Rehabilitation;9:34-37.

21. Zhang L, Zhao M, Sui RB.Cerebellar Fastigial Nucleus Electrical Stimulation Alleviates Depressive-Like Behaviors in Post-Stroke Depression Rat Model and Potential Mechanisms.Cell Physiol Biochem. 2017;41:1403-1412. Doi: 10.1159/000467940.

\section{Tables}

Table 1 Comparison of baseline characteristics at admission between patients with Sham and FNS groups. 


\begin{tabular}{|c|c|c|c|c|}
\hline & Sham group (133) & PMES group (125) & OR(95\%CI) & $\mathrm{P} *$ \\
\hline Age, y(Mean SD) & $66.11 \pm 8.37$ & $65.0 \pm 8.82$ & & 0.622 \\
\hline NIHSS, (Mean SD) & $6.99 \pm 2.47$ & $7.02 \pm 2.21$ & & 0.978 \\
\hline Females, n(\%) & $68(51.13)$ & $55(44.00)$ & $0.751(0.46-1.23)$ & 0.465 \\
\hline Men, n(\%) & $65(48.87)$ & $70(56.00)$ & $0.751(0.46-1.23)$ & 0.252 \\
\hline $\mathrm{BMI} \geq 24 \mathrm{~kg} / \mathrm{m}, \mathrm{n}(\%)$ & $32(24.06)$ & $42(33.60)$ & $1.60(0.93-2.75)$ & 0.090 \\
\hline Hypertension, n(\%) & $72(54.14)$ & $76(60.8)$ & $1.31(0.80-2.16)$ & 0.279 \\
\hline Current Smoking, n(\%) & $48(36.09)$ & $43(34.40)$ & $0.93(0.56-1.55)$ & 0.776 \\
\hline Current Drinking, n(\%) & $43(32.33)$ & $44(33.08)$ & $1.14(0.68-1.91)$ & 0.626 \\
\hline Diabetes, n(\%) & $54 \llbracket 40.60 \square$ & $43(34.40)$ & $0.77(0.46-1.27)$ & 0.304 \\
\hline Hyperlipidemia, n(\%) & $65(48.87)$ & $74(59.20)$ & $1.52(0.93-2.45)$ & 0.096 \\
\hline Atrial fibrillation, n(\%) & $50(37.59)$ & $40(30.08)$ & $0.78(0.47-1.31)$ & 0.346 \\
\hline Family history of stroke, n(\%) & $29(21.80)$ & $33(26.40)$ & $1.29(0.73-2.28)$ & 0.388 \\
\hline MoCA Score, (mean SD) & $24.90 \pm 3.16$ & $24.90 \pm 2.82$ & & 0.936 \\
\hline HRSD Score, (mean SD) & $22.02 \pm 4.54$ & $21.51 \pm 4.32$ & & 0.280 \\
\hline \multicolumn{5}{|l|}{ Medications use } \\
\hline Antiplatelet, n(\%) & $43(32.33)$ & $48(38.40)$ & 1.31匹0.78-2.17凸 & 0.308 \\
\hline Antihypertensive, n(\%) & $56(42.11)$ & $56(44.80)$ & $1.12(0.68-1.83)$ & 0.663 \\
\hline lipid-lowering medications, n(\%) & $64(48.12)$ & $71(56.80)$ & $1.42 \llbracket 0.87-2.32 \square$ & 0.163 \\
\hline Sertraline, n(\%) & $83(62.41)$ & $81(60.90)$ & $1.11(0.67-1.84)$ & 0.690 \\
\hline Citalopram, n(\%) & $14(10.53)$ & 19(14.29) & $1.52(0.73-3.19)$ & 0.261 \\
\hline $\begin{array}{l}\text { Paroxetine, } \mathrm{n}(\%) \\
\text { Infarct location }\end{array}$ & $36(27.07)$ & $25(18.80)$ & $0.67(0.38-1.21)$ & 0.182 \\
\hline Basal ganglia, n(\%) & $62(46.62)$ & $61(45.86)$ & $1.09(0.67-1.78)$ & 0.726 \\
\hline Brain stem, n(\%) & $18(13.53)$ & $20(15.04)$ & $1.22(0.611-2.43)$ & 0.576 \\
\hline Cerebellum, n(\%) & $10(7.52)$ & $4(3.01)$ & $0.41(0.12-1.33)$ & 0.126 \\
\hline Frontal lobe, n(\%) & $19(14.29)$ & $15(11.28)$ & $0.82(0.40-1.69)$ & 0.588 \\
\hline Parietal lobe, n(\%) & $10(7.52)$ & $9(6.77)$ & $0.95 \square 0.37-2.43 \square$ & 0.922 \\
\hline Temporal lobe, n(\%) & $5(3.76)$ & $10(8.00)$ & $2.23 \sqcap 0.74-6.71 \square$ & 0.146 \\
\hline Occipital lobe, n(\%) & $9(6.77)$ & $6(4.80)$ & $0.70 \sqcap 0.24-2.01 \square$ & 0.500 \\
\hline
\end{tabular}

BMI:Body Mass Index .SD: standard deviation

*Comparison between sham and PMES groups. Demographic characteristics were compared between the 2 subgroups in univariate analysis, using Pearson $\chi 2$ test, Fisher exact 2-sided test, or Student t test, mean values( \pm standard deviation) were calculated for continuous variables. Mann-Whitney U test was used to test differences between two group.

Table 2 The mean value of the MoCA Score and HRSD at 6 months in Sham and PMES groups 


\begin{tabular}{llll}
\hline & \multicolumn{1}{c}{ Sham group } & PMES group & $\mathrm{P}^{*}$ \\
\hline MoCA Score, (Mean SD) & $27.26 \pm 2.20$ & $28.26 \pm 1.95$ & 0.000 \\
HRSD Score, (Mean SD) & $11.54 \pm 4.21$ & $9.58 \pm 3.45$ & 0.000 \\
\hline
\end{tabular}

SD: standard deviation

*Continuous variables are expressed as mean \pm standard deviation. Mann-Whitney U test was used to test differences between two groups.

Table 3 Multivariable Models Showing Association between PMES and poststroke depression

\begin{tabular}{lrc}
\hline & OR $(95 \% \mathrm{CI})$ & $\mathrm{P}^{*}$ \\
\hline Model 1 (Baseline MoCA) & & \\
Treatment response $(\geq 50 \%$ reduction in HRSD) & $2.04(1.15-3.63)$ & 0.015 \\
Depression remission (HRSD $\leq 9)$ & $1.83(1.05-3.18)$ & 0.032 \\
Model 2 (6-month MoCA) & & \\
Treatment response ( $\geq 50 \%$ reduction in HRSD) & $2.12(1.17-3.86)$ & 0.013 \\
Depression remission (HRSD $\leq 9)$ & $1.70(0.94-3.06)$ & 0.047 \\
\hline
\end{tabular}

*Multivariable adjusted for age, NIHSS, sex, BMI $\square$ hypertension, current smoking, current drinking, diabetes, hyperlipidemia, atrial fibrillation, family history of stroke,baseline MoCA score, HRSD score, medications use, infarct location

\section{Figures}




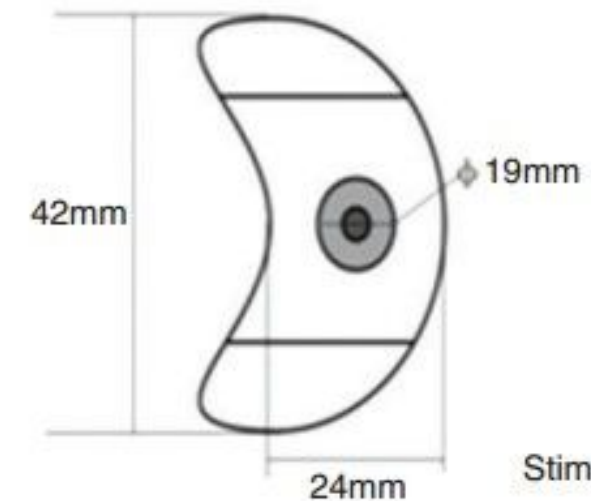

Stimulation electrode
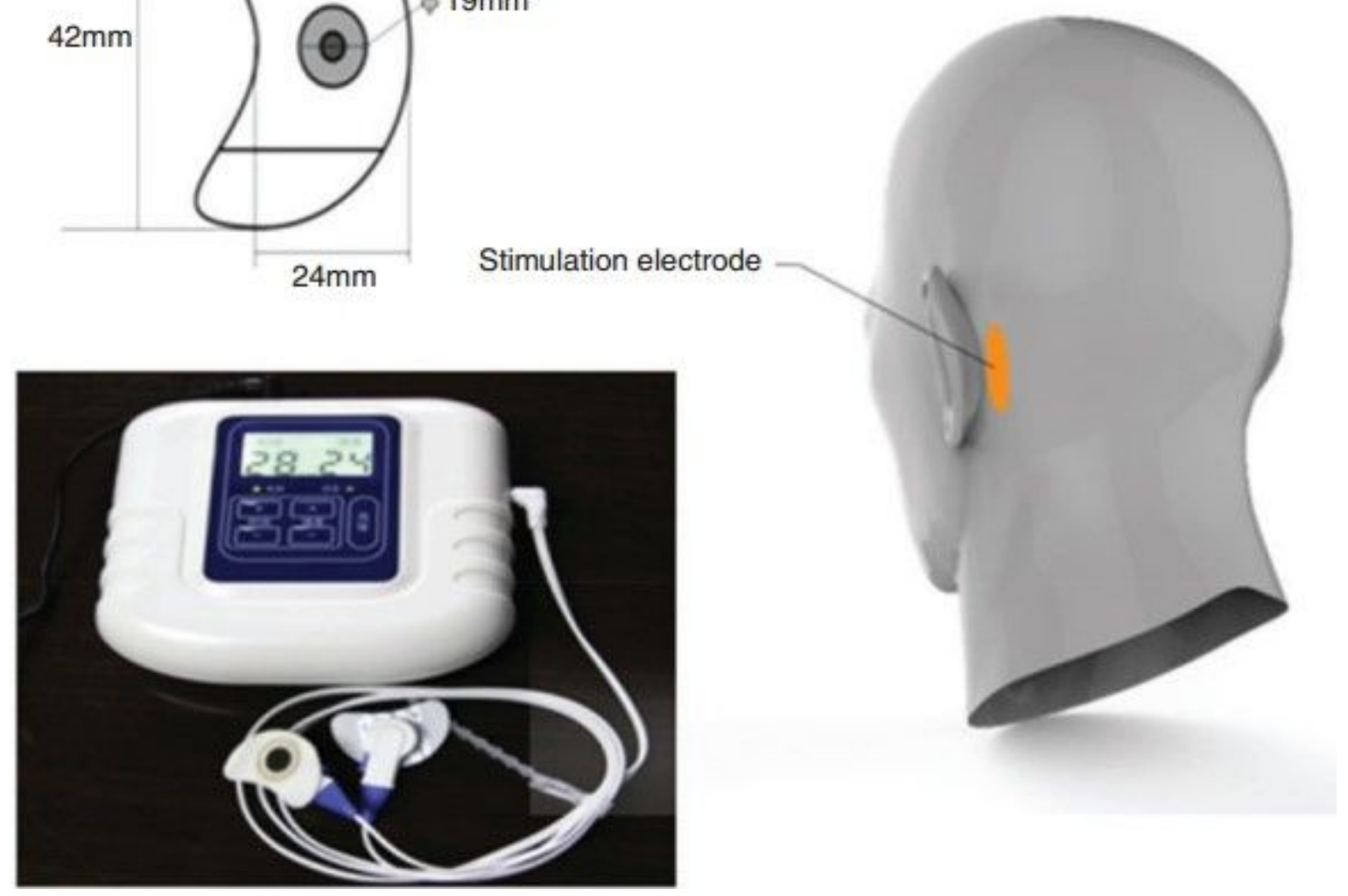

\section{Figure 1}

The percutaneous mastoid electrical stimulator (PMES) device and stimulation electrode placed on mastoid area behind the ear 